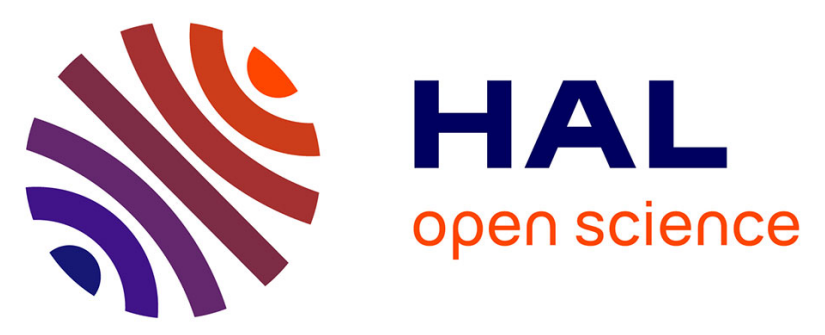

\title{
Am and pm noise analysis in quartz crystal oscillators: symbolic calculus approach
}

Nicolas Ratier, Laurent Couteleau, Rémi Brendel, Philippe Guillemot

\section{To cite this version:}

Nicolas Ratier, Laurent Couteleau, Rémi Brendel, Philippe Guillemot. Am and pm noise analysis in quartz crystal oscillators: symbolic calculus approach. International Frequency Control Symposium (IEEE), May 1998, Pasadena, California, United States. pp.156-163. hal-00345179

\author{
HAL Id: hal-00345179 \\ https://hal.science/hal-00345179
}

Submitted on 8 Dec 2008

HAL is a multi-disciplinary open access archive for the deposit and dissemination of scientific research documents, whether they are published or not. The documents may come from teaching and research institutions in France or abroad, or from public or private research centers.
L'archive ouverte pluridisciplinaire $\mathbf{H A L}$, est destinée au dépôt et à la diffusion de documents scientifiques de niveau recherche, publiés ou non, émanant des établissements d'enseignement et de recherche français ou étrangers, des laboratoires publics ou privés. 


\title{
AM AND PM NOISE ANALYSIS IN QUARTZ CRYSTAL OSCILLATORS: SYMBOLIC CALCULUS APPROACH
}

\author{
N. Ratier, L. Couteleau, R. Brendel, P. Guillemot $\dagger$ \\ Laboratoire de Physique et Métrologie des Oscillateurs du C.N.R.S. \\ associé à l'Université de Franche-Comté-Besançon \\ 32 avenue de l'Observatoire - 25044 Besançon Cedex - France \\ nratier@lpmo.univ-fcomte.fr \\ †Centre National d'Etudes Spatiales \\ 18 avenue E. Belin - 31055 Toulouse Cedex - France
}

\begin{abstract}
Increasing performance of quartz crystal oscillators as well as predictability requirements when developing the devices need accurate analysis of noise sources. Our work is devoted to understand how an oscillator reacts to additive noise of an element in the electronic circuit. Up to now, oscillator designers often refer to the well-known Leeson's model to explain the shape of phase noise spectral density. This physical model only allows one to obtain the global phase noise spectrum. By considering each noise source individually, we can obtain the comparative contribution of the sources. Then AM and PM noise source spectra can be related to the circuit architecture.

The influence of an individual noise source can be obtained from the differential equation describing the oscillator behavior. Nevertheless, setup of the differential equation from the inspection of the circuit involves lengthy and tedious algebraic calculations almost impossible to achieve by hand. By using symbolic calculation capability of formal calculus programs, it is possible to automatically derive the differential equation of the oscillator including noise sources from a SPICE netlist description of the circuit. The resulting expressions can be edited under the form of high level language code (Fortran, C, ...) which is eventually compiled and linked with the numerical programs calculating the noise spectra.

This paper presents the method to construct the differential equations in a fully automatic way regardless of the studied oscillator circuit.
\end{abstract}

\section{Introduction}

Equation derivation of the differential equation of the oscillator behavior, including noise source is very tedious, and involves lengthy calculations. It is carried out in four distinct steps summarized below:

- The first step of the process is to reduce the electronic circuit of the oscillator, including individual noise sources, to a canonical form. This step is the only one which is dependent of the studied oscillator. The three next steps are done once and for all.

- The second step is to obtain the system of equations governing the oscillator behavior from the canonical form of the circuit. This system represents the oscillation condition.

- The third step concerns the algebraic resolution of the previous system. A straightforward demonstration to prove the general form is proposed.

- The fourth step presents the method used to transform the initial ODE under the form of an integrodifferential equation suitable for the use of an asymptotic pertubation method.

This paper is devoted to the algorithmic construction of this ODE, valid for one transistor oscillator.

\section{First step: Reduction to a canonical form}

We consider a quartz oscillator circuit with one transistor as, shown for example, in figure (1). The additive noise sources of the circuit components are represented by a current generator. 


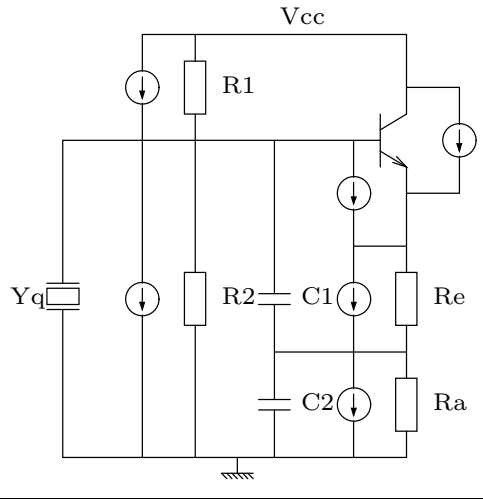

Figure 1. Oscillator circuit

The transistor is represented [4] by the large signal y-parameter equivalent circuit shown in figure (2). The 4 admittances $Y_{i}, Y_{r}, Y_{f}, Y_{o}$ permit to modelize the nonlinear behavior of the component. Their value depend on the input voltage $u$, on the output voltage $v$ and on the pulsation $\omega(s=j \omega)$.

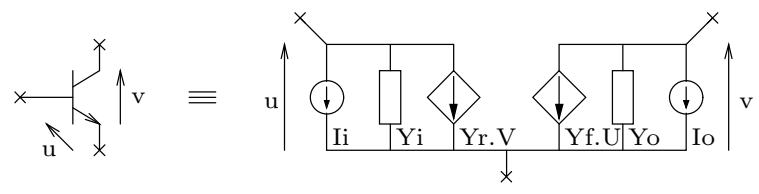

Figure 2. y-parameter representation of the transistor

The admittances $Y_{i}, Y_{r}, Y_{f}, Y_{o}$ can be expressed under the form (1), functions $G_{i}, C_{i}, G_{r}, C_{r}, G_{f}, C_{f}, G_{o}, C_{o}$ are calculated [1] for a given bias and temperature condition by using the electrical simulator SPICE [5] [9], they are available under the form of a table.

$$
\begin{aligned}
Y_{i}(|u|) & =G_{i}(|u|)+s C_{i}(|u|) \\
Y_{r}(|v|) & =G_{r}(|v|)+s C_{r}(|v|) \\
Y_{f}(|u|) & =G_{f}(|u|)+s C_{f}(|u|) \\
Y_{o}(|v|) & =G_{o}(|v|)+s C_{o}(|v|)
\end{aligned}
$$

The equivalent noise sources $I_{i}, I_{o}$ modelize the noise of the transistor. Thermal noise source mean square current is given by (2a), where $k$ is the Boltzmann's constant, $T$ the absolute temperature, $G_{i}$ (or $G_{o}$ ) the component conductance, $q$ the electron charge, $I_{c}$ the DC collector current and $\Delta f$ the effective bandwith. Voltages $u$ and $v$ are calculated in the steady state without noise.

$$
\begin{aligned}
& \overline{I_{i}^{2}}=4 k T G_{i}(|u|) \Delta f+2 q I_{c} \Delta f \\
& \overline{I_{o}^{2}}=4 k T G_{o}(|v|) \Delta f+2 q I_{c} \Delta f
\end{aligned}
$$

Basically the method consists in reducing the oscillator circuit to an equivalent canonical circuit whose calculus can be done once and for all.

After grounding the supply voltage $V c c$, the reduction process starts by performing all the elementary transforms like series or parallel associations of admittances. Then, the electronic circuit is progressively reduced by using recursively either one of the 6 transformations represented in Fig. 3. At each step of the reduction process, a new circuit component is $C_{o}$ introduced into the amplifier. The initial amplifier is the transistor itself (see Fig. 2).

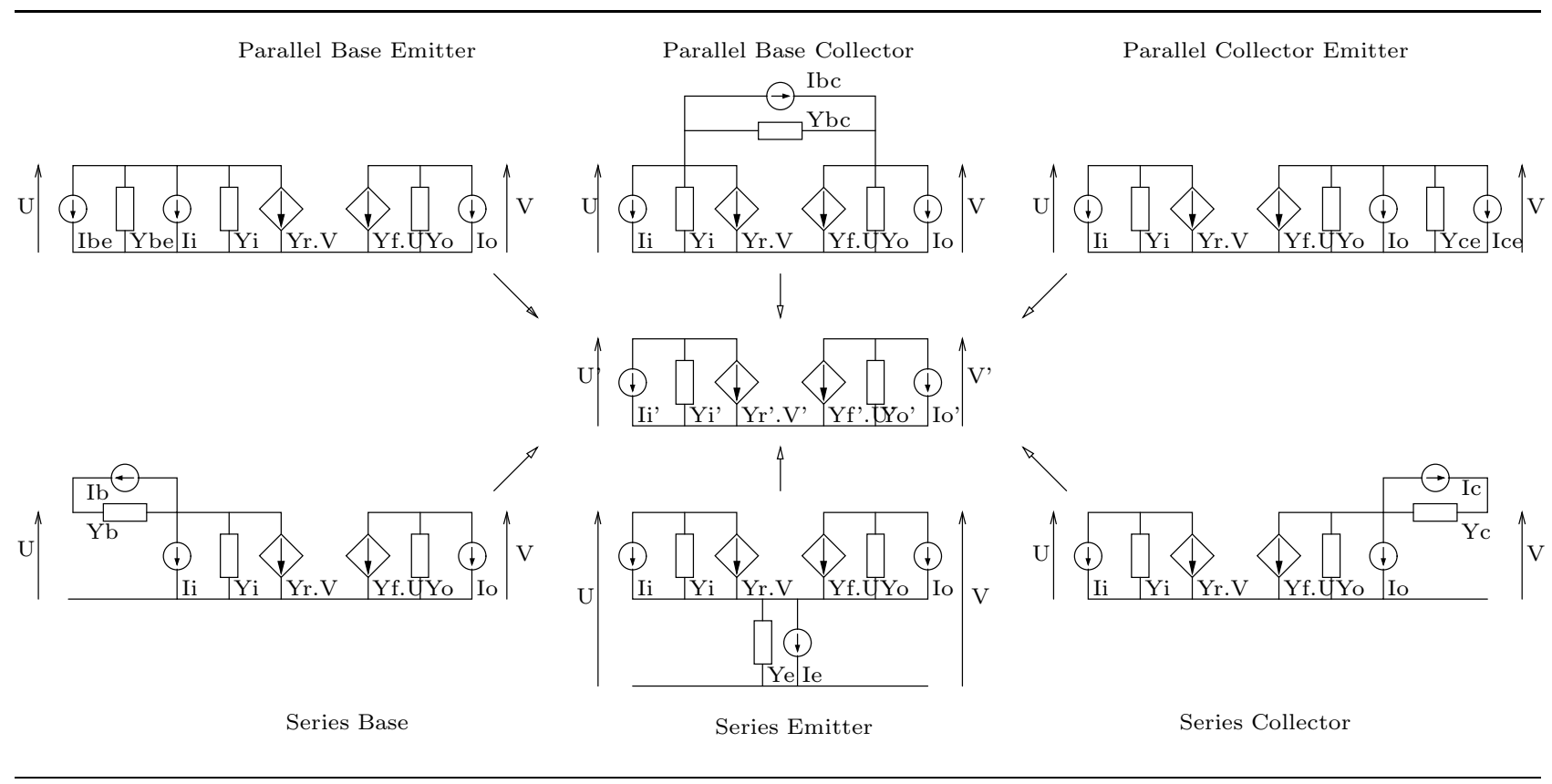

Figure 3. The 6 transformations for circuit reduction 
- The $Y$-parameters $Y_{i}^{\prime}, Y_{r}^{\prime}, Y_{f}^{\prime}, Y_{o}^{\prime}$ of the new equivalent circuit are expressed in terms of the former ones $Y_{i}, Y_{r}, Y_{f}, Y_{o}$ and the admittance to be introduced (Table 1). The initial $Y$-parameters are the nonlinear representation of the transistor (Equ. 1).

Table 1. Admittance equations

\begin{tabular}{|c|c|}
\hline & $Y_{i}, Y_{r}, Y_{f}, Y_{o}$ \\
\hline $\begin{array}{l}\text { Parallel } \\
\text { Base-Emitter }\end{array}$ & $\begin{array}{l}Y_{i}^{\prime}=Y_{i}+Y_{b e} \\
Y_{r}^{\prime}=Y_{r} \\
Y_{f}^{\prime}=Y_{f} \\
Y_{o}^{\prime}=Y_{o}\end{array}$ \\
\hline $\begin{array}{l}\text { Parallel } \\
\text { Collector-Emitter }\end{array}$ & $\begin{aligned} Y_{i}^{\prime} & =Y_{i} \\
Y_{r}^{\prime} & =Y_{r} \\
Y_{f}^{\prime} & =Y_{f} \\
Y_{o}^{\prime} & =Y_{o}+Y_{c e}\end{aligned}$ \\
\hline $\begin{array}{l}\text { Parallel } \\
\text { Base-Collector }\end{array}$ & $\begin{array}{l}Y_{i}^{\prime}=Y_{i}+Y_{b c} \\
Y_{r}^{\prime}=Y_{r}-Y_{b c} \\
Y_{f}^{\prime}=Y_{f}-Y_{b c} \\
Y_{o}^{\prime}=Y_{o}+Y_{b c}\end{array}$ \\
\hline $\begin{array}{l}\text { Series } \\
\text { Base }\end{array}$ & $\begin{array}{l}Y_{i}^{\prime}=\frac{Y_{i} Y_{b}}{Y_{i}+Y_{b}} \\
Y_{r}^{\prime}=\frac{Y_{r} Y_{b}}{Y_{i}+Y_{b}} \\
Y_{f}^{\prime}=\frac{Y_{f} Y_{b}}{Y_{i}+Y_{b}} \\
Y_{o}^{\prime}=\frac{Y_{o} Y_{b}+\left(Y_{i} Y_{o}-Y_{r} Y_{f}\right)}{Y_{i}+Y_{b}}\end{array}$ \\
\hline $\begin{array}{l}\text { Series } \\
\text { Collector }\end{array}$ & $\begin{array}{l}Y_{i}^{\prime}=\frac{Y_{i} Y_{c}+\left(Y_{i} Y_{o}-Y_{r} Y_{f}\right)}{Y_{o}+Y_{c}} \\
Y_{r}^{\prime}=\frac{Y_{r} Y_{c}}{Y_{Y}+Y_{c}} \\
Y_{f}^{\prime}=\frac{Y_{f} Y_{c}}{Y_{O}+Y_{c}} \\
Y_{o}^{\prime}=\frac{Y_{o} Y_{c}}{Y_{o}+Y_{c}}\end{array}$ \\
\hline $\begin{array}{l}\text { Series } \\
\text { Emitter }\end{array}$ & $\begin{aligned} Y_{i}^{\prime} & =\frac{Y_{i}+Y_{e}+\left(Y_{i} Y_{o}-Y_{r} Y_{f}\right)}{Y_{i}+Y_{r}+Y_{f}+Y_{o}+Y_{e}} \\
Y_{r}^{\prime} & =\frac{Y_{r} Y_{e}-\left(Y_{i} Y_{o}-Y_{r} Y_{f}\right)}{Y_{i}+Y_{Y}+Y_{f}+Y_{+}+Y_{e}} \\
Y_{f}^{\prime} & =\frac{Y_{f} Y_{e}-\left(Y_{i} Y_{o}-Y_{r} Y_{f}\right)}{Y_{i}+Y_{r}+Y_{f}+Y_{o}+Y_{e}} \\
Y_{o}^{\prime} & =\frac{Y_{o} Y_{e}+\left(Y_{i} Y_{o}-Y_{r} Y_{f}\right)}{Y_{i}+Y_{r}+Y_{f}+Y_{o}+Y_{e}}\end{aligned}$ \\
\hline
\end{tabular}

- The input and output noise sources $I_{i}^{\prime}, I_{o}^{\prime}$ are expressed in terms of the former ones $I_{i}, I_{o}$ (Table 2). The initial noise current source is the noise of the transistor (Equ. 2).

Table 2. Noise equations

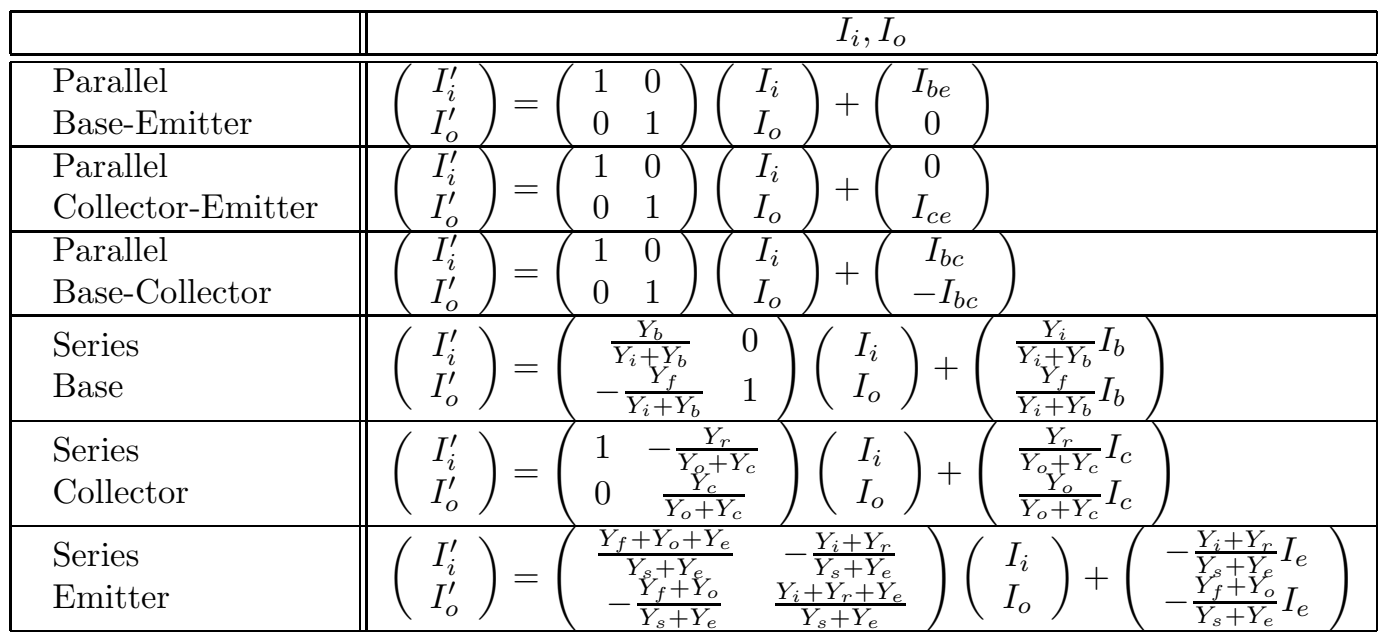


- The input and output voltages $U^{\prime}, V^{\prime}$ are expressed in terms of the former ones $U, V$. (Table 3). The initial voltage are the voltage $(u, v)$ across the transistor itself (Fig. 2).

Table 3. Voltage equations

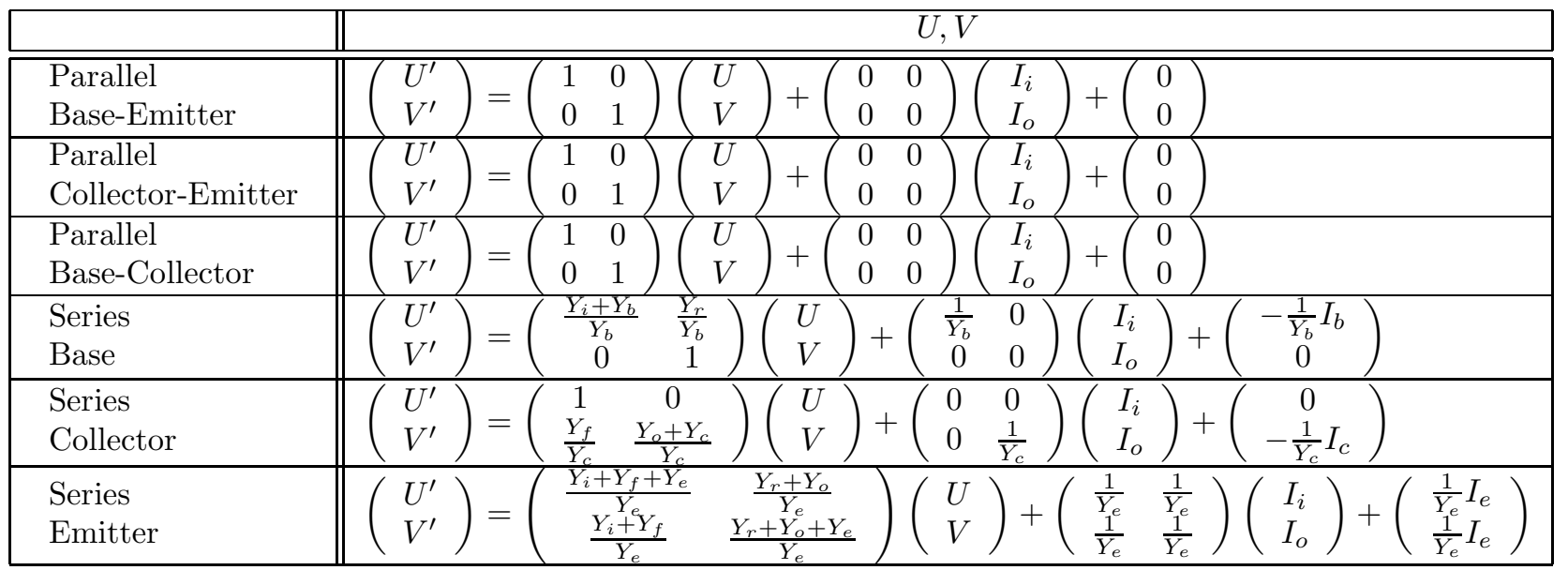
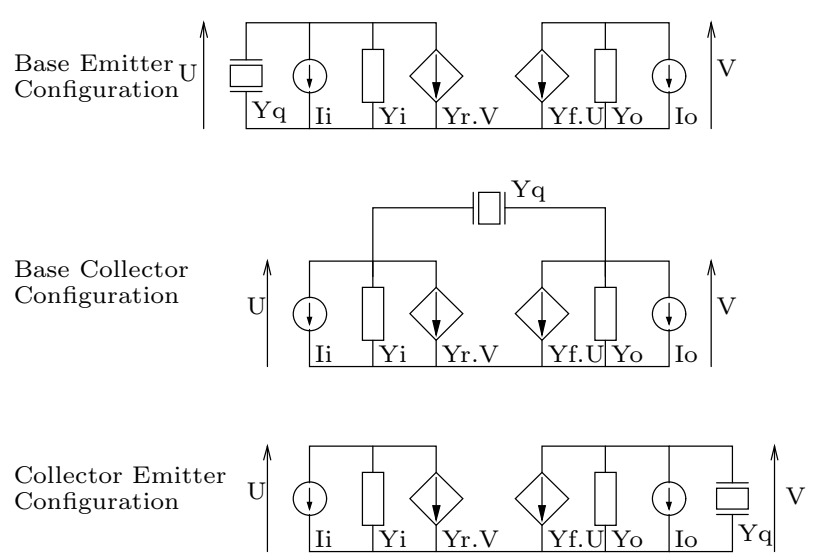

Figure 4. Reduced forms of an oscillator
The reduction process go on until no more $R L C$ admittance remains outside of the amplifier circuit. When the reduction process is completed, the resonator may occupy one of the three basic configurations shown in figure (4).

\section{Second step: Oscillation condition}

Assuming that $n$ reduction steps were needed to reduce the circuit, the application of Kirchhoff's law to both input and output parts of each of the 3 circuits in figure (4) leads to equation (3). According to the configuration, the coefficients $\left\{Q_{11}, Q_{12}, Q_{21}, Q_{22}\right\}$ are equal either to 0 or 1 . :

$$
\begin{aligned}
& {\left[\left(\begin{array}{cc}
Y_{i}^{(n)} & Y_{r}^{(n)} \\
Y_{f}^{(n)} & Y_{o}^{(n)}
\end{array}\right)+Y_{q}\left(\begin{array}{ll}
Q_{11} & Q_{12} \\
Q_{21} & Q_{22}
\end{array}\right)\right]\left(\begin{array}{c}
U^{(n)} \\
V^{(n)}
\end{array}\right)=-\left(\begin{array}{c}
I_{i}^{(n)} \\
I_{o}^{(n)}
\end{array}\right)} \\
& \text { with }\left(\begin{array}{ll}
Q_{11} & Q_{12} \\
Q_{21} & Q_{22}
\end{array}\right)=\left(\begin{array}{ll}
1 & 0 \\
0 & 0
\end{array}\right) \quad \text { Base Emitter } \\
& =\left(\begin{array}{rr}
1 & -1 \\
-1 & 1
\end{array}\right) \quad \text { Base Collector } \\
& =\left(\begin{array}{ll}
0 & 0 \\
0 & 1
\end{array}\right) \quad \text { Collector Emitter }
\end{aligned}
$$

Remember that the three systems of (3) are not linear because the transistor $Y$-parameters depend on the input or output voltages $u$ or $v$.

The previous equation (3) involves reduced noise sources $\left(I_{i}^{(n)}, I_{o}^{(n)}\right)$ and voltages $\left(U^{(n)}, V^{(n)}\right)$ at the final step of the reduction process. To determine the oscillation condition equation, one must express the noise $\left(I_{i}^{(n)}, I_{o}^{(n)}\right)$ and the voltage $\left(U^{(n)}, V^{(n)}\right)$ in terms of the voltage $(u, v)$ across the transistor (Fig. 2 ) and the noise current of each circuit admittance (Fig. 1). 
Tables (2) and (3) show that the equation built during the reduction process has the form shown by Eq. (4, 5). We will show, by recurrence, that they can be written under the form $(6,7)$. The $j$ indice is summed over $\{i, o, 1, \ldots, n\}$, the letters $i$ and $o$ (resp. In et Out) represent the transistor noise and the numbers 1 to $n$ represent the component noise (possibly null in the case of noiseless component).

$$
\begin{aligned}
& \left(\begin{array}{c}
I_{i}^{(n)} \\
I_{o}^{(n)}
\end{array}\right)=\left(\begin{array}{ll}
A_{11}^{(n)} & A_{12}^{(n)} \\
A_{21}^{(n)} & A_{22}^{(n)}
\end{array}\right)\left(\begin{array}{c}
I_{i}^{(n-1)} \\
I_{o}^{(n-1)}
\end{array}\right)+\left(\begin{array}{c}
B_{1}^{(n)} \\
B_{2}^{(n)}
\end{array}\right) I_{n} \\
& \left(\begin{array}{l}
U^{(n)} \\
V^{(n)}
\end{array}\right)=\left(\begin{array}{ll}
C_{11}^{(n)} & C_{12}^{(n)} \\
C_{21}^{(n)} & C_{22}^{(n)}
\end{array}\right)\left(\begin{array}{c}
U^{(n-1)} \\
V^{(n-1)}
\end{array}\right)+\left(\begin{array}{cc}
D_{11}^{(n)} & D_{12}^{(n)} \\
D_{21}^{(n)} & D_{22}^{(n)}
\end{array}\right)\left(\begin{array}{c}
I_{i}^{(n-1)} \\
I_{o}^{(n-1)}
\end{array}\right)+\left(\begin{array}{c}
E_{1}^{(n)} \\
E_{2}^{(n)}
\end{array}\right) I_{n}
\end{aligned}
$$

The form used in Eq. (6), allows one to replace the recurrence relation using $\left(I_{i}^{(n)}, I_{o}^{(n)}\right)$ and $\left(U^{(n)}, V^{(n)}\right)$ by a recurrence relation using the co- efficient $K_{j}^{(n)}, F^{(n)}, L_{j}^{(n)}$. Thes equations expresses $\left(I_{i}^{(n)}, I_{o}^{(n)}\right)$ and $\left(U^{(n)}, V^{(n)}\right)$ directly in terms of $(u, v)$ and $I_{j}$.

$$
\begin{aligned}
\left(\begin{array}{c}
I_{i}^{(n)} \\
I_{o}^{(n)}
\end{array}\right) & =\sum_{j}^{\{i, o, 1, \ldots, n\}}\left(\begin{array}{c}
K_{1 j}^{(n)} \\
K_{2 j}^{(n)}
\end{array}\right) I_{j} \\
\left(\begin{array}{c}
U^{(n)} \\
V^{(n)}
\end{array}\right) & =\left(\begin{array}{ll}
F_{11}^{(n)} & F_{12}^{(n)} \\
F_{21}^{(n)} & F_{22}^{(n)}
\end{array}\right)\left(\begin{array}{c}
u \\
v
\end{array}\right)+\sum_{j}^{\{i, o, 1, \ldots, n\}}\left(\begin{array}{c}
L_{1 j}^{(n)} \\
L_{2 j}^{(n)}
\end{array}\right) I_{j}
\end{aligned}
$$

\section{Third step: Transient state ODE}

The previous calculus allowed us to obtain the system $(3,6,7)$ which expresses the voltages $u$ and $v$ in terms of the circuit component and their associated noise source. We will show now that the resolution of these equations with respect to $u$ and $v$ leads to equations $(8,9)$.

$$
\begin{aligned}
u & =\frac{\sum_{j}\left(C_{j}+D_{j} Y_{q}\right) I_{j}}{A+B Y_{q}} \\
v & =\frac{\sum_{j}\left(E_{j}+F_{j} Y_{q}\right) I_{j}}{A+B Y_{q}}
\end{aligned}
$$

\section{Proof}

The resolution of equations $(3,6,7)$ involves only simple algebraic operations. The calculus, and in particular the proof that there is no term in $Y_{q}^{2}$ require about 5 hand written pages.

The proof can be done by using computer algebra system (CAS like Maple V.4, Mathematica, Reduce, ...).

\section{Fourth step: Transient calculation ODE}

The previous section showed that the oscillation condition has the general form $(10,11)$. The factor $A, B, C_{j}, D_{j}, E_{j}, F_{j}$ are rational functions in the Laplace's variable $s$ and $Y_{q}$ is the admittance of the resonator.

$$
\begin{aligned}
\left(A+B Y_{q}\right) u & =\sum_{j}\left(C_{j}+D_{j} Y_{q}\right) I_{j} \\
\left(A+B Y_{q}\right) v & =\sum_{j}\left(E_{j}+F_{j} Y_{q}\right) I_{j}
\end{aligned}
$$

The nonlinear ordinary differential equation given the oscillation signal can be obtained by replacing the Laplace's variable $s$ by the differential operator $d / d t$ in Equ. $(10,11)$. Then, this ODE could be solved by using usual numerical methods. Unfortunately, these algorithms generally fail to converge in a reasonable computing time because of the high quality factor of the quartz oscillator circuits.

However, the previous ODE can be solved by using perturbation methods dealing with equations of the form $\ddot{u}+\omega_{0}^{2} u=f(x, \dot{x}, x)$, with a "small" perturbation function $f$ at the right hand side. This method is well suited to quartz oscillator analysis because: it is well known that the higher the quality factor the closer the output signal to a sinusoid whose frequency is determined by the motional part 
of the resonator, i.e. $f_{q}=1 /\left(2 \pi \sqrt{L_{q q} C_{q q}}\right)$. The algebraic manipulations have now to put the previous ODE under the form of a second order linear harmonic differential equation (i.e. $\ddot{u}+\omega_{q}^{2} u$ ) and a small nonlinear integrodifferential right hand side.

The resonator is modeled by the classical equivalent circuit shown in Fig. 5.

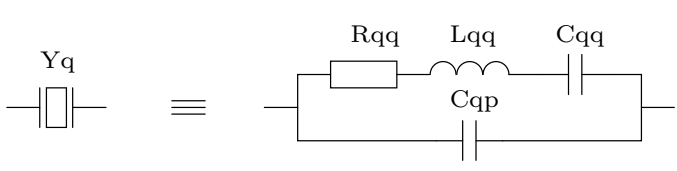
12.

$$
Y_{q}=s C_{q p}+\frac{s / L_{q q}}{s^{2}+\frac{R_{q q}}{L_{q q}} s+\omega_{q}^{2}}, \omega_{q}^{2}=\frac{1}{L_{q q} C_{q q}}
$$

We will show that the oscillator equation $(10,11)$ can be written under the form (13), where the amplitude of $f$ is small. This allows to obtain the ODE under a form suitable for the perturbation method.

$$
\ddot{u}+\omega_{0}^{2} u=f\left(\ldots, \iint u d t^{2}, \int u d t, u, \frac{d u}{d t}, \frac{d^{2} u}{d t^{2}} \ldots\right)
$$

\section{Proof}

The oscillation condition has the form

$$
\left\{\begin{aligned}
\left(A+B Y_{q}\right) u & =\sum_{j}\left(C_{j}+D_{j} Y_{q}\right) I_{j} \\
\left(A+B Y_{q}\right) v & =\sum_{j}\left(E_{j}+F_{j} Y_{q}\right) I_{j}
\end{aligned}\right.
$$

By replacing the expression of $Y_{q}$ given by Equ. (12) one obtains:

$$
\begin{aligned}
\left(s^{2}+\frac{R_{q q}}{L_{q q}} s+\omega_{q}^{2}\right) u & =-\frac{B p / L_{q q}}{\left(A+B s C_{q p}\right)} u \\
& +\sum_{j} \frac{\left(C_{j}+D_{j} Y_{q}\right)}{\left(A+B s C_{q p}\right)} I_{j} \\
\left(s^{2}+\frac{R_{q q}}{L_{q q}} s+\omega_{q}^{2}\right) v & =-\frac{B p / L_{q q}}{\left(A+B s C_{q p}\right)} v \\
& +\sum_{j} \frac{\left(E_{j}+F_{j} Y_{q}\right)}{\left(A+B s C_{q p}\right)} I_{j}
\end{aligned}
$$

Remember that $A, B, C_{j}, D_{j}, E_{j}, F_{j}$ are rational functions of the Laplace's variable $s$. Let $D(s)$ be the common denominator of Equ. (16), $j$ vary from 1 to $n$.

$$
\frac{B s / L_{q q}}{\left(A+B s C_{q p}\right)}, \frac{C_{j}}{\left(A+B s C_{q p}\right)}, \frac{D_{j}}{\left(A+B s C_{q p}\right)}
$$

Let

$$
\begin{aligned}
& N_{1}(s) \text { the polynomial } \frac{D(s) B s / L_{q q}}{\left(A+B s C_{q p}\right)} \\
& N_{2 j}(s) \text { the polynomial } \frac{D(s) C_{j}}{\left(A+B s C_{q p}\right)} \\
& N_{3 j}(s) \text { the polynomial } \frac{D(s) D_{j}}{\left(A+B s C_{q p}\right)}
\end{aligned}
$$

Likewise, let $D^{\prime}(s)$ be the common denominator of Equ. (18), $j$ vary from 1 to $n$.

$$
\frac{B s / L_{q q}}{\left(A+B s C_{q p}\right)}, \frac{E_{j}}{\left(A+B s C_{q p}\right)}, \frac{F_{j}}{\left(A+B s C_{q p}\right)}
$$

Let

$$
\begin{gathered}
N_{1}^{\prime}(s) \text { the polynomial } \frac{D^{\prime}(s) B s / L_{q q}}{\left(A+B s C_{q p}\right)} \\
N_{2 j}^{\prime}(s) \text { the polynomial } \frac{D^{\prime}(s) E_{j}}{\left(A+B s C_{q p}\right)} \\
N_{3 j}^{\prime}(s) \text { the polynomial } \frac{D^{\prime}(s) F_{j}}{\left(A+B s C_{q p}\right)}
\end{gathered}
$$

Despite their appearence, Equ. (17) and (19) are no longer rational functions but polynomials because of the definition of $D(s)$ and $D^{\prime}(s)$. The previous definitions leads to equations (20).

$$
\begin{aligned}
\left(s^{2}+\frac{R_{q q}}{L_{q q}} s+\omega_{q}^{2}\right) u & =-\frac{N_{1}(s)}{D(s)} u \\
& +\sum_{j} \frac{N_{2 j}(s)+N_{3 j}(s) Y_{q}}{D(s)} I_{j} \\
\left(s^{2}+\frac{R_{q q}}{L_{q q}} s+\omega_{q}^{2}\right) v & =-\frac{N_{1}^{\prime}(s)}{D^{\prime}(s)} v \\
& +\sum_{j} \frac{N_{2 j}^{\prime}(s)+N_{3 j}^{\prime}(s) Y_{q}}{D(s)} I_{j}
\end{aligned}
$$

For the perturbation method to be applicable, the right hand side of equation (20) must be small. To this end, one must identify the dominating term $D_{k} s^{k}$ of the denominator $D(s)$ and the dominating term of the denominator $D^{\prime}(s)$ which can be written under the form $(21,22)$.

$$
\begin{aligned}
D(s) & =D_{k} s^{k}+\sum_{\substack{i=0 \\
i \neq k}}^{\operatorname{deg}(D)} D_{i} s^{i} \\
D^{\prime}(s) & =D_{k}^{\prime} s^{k}+\sum_{\substack{i=0 \\
i \neq k}}^{\operatorname{deg}(D)} D_{i}^{\prime} s^{i}
\end{aligned}
$$

In practice, the modulus of each term in the sums $(21,22)$ is computed in the steady state of the noiseless oscillator [2][7][8]. Then, the equation (20) takes the form (23): 


$$
\left\{\begin{array}{l}
\left(s^{2}+\frac{R_{q q}}{L_{q q}} s+\omega_{q}^{2}\right)\left(D_{k} s^{k}+\sum_{\substack{i=0 \\
i \neq k}}^{\operatorname{deg}(D)} D_{i} s^{i}\right) u=-N_{1}(s) u+\sum_{j}\left(N_{2 j}(s)+N_{3 j}(s) Y_{q}\right) I_{j} \\
\left(s^{2}+\frac{R_{q q}}{L_{q q}} s+\omega_{q}^{2}\right)\left(D_{k}^{\prime} s^{k}+\sum_{\substack{i=0 \\
i \neq 0 \\
\operatorname{deg}\left(D^{\prime}\right)}} D_{i}^{\prime} s^{i}\right) v=-N_{1}^{\prime}(s) v+\sum_{j}\left(N_{2 j}^{\prime}(s)+N_{3 j}^{\prime}(s) Y_{q}\right) I_{j}
\end{array}\right.
$$

System (23) can be written under the form (24).

$$
\left\{\begin{array}{l}
\left(s^{2}+\frac{R_{q q}}{L_{q q}} s+\omega_{q}^{2}\right) u=-\left(s^{2}+\frac{R_{q q}}{L_{q q}} s+\omega_{q}^{2}\right) \frac{\sum_{\substack{i=0 \\
i \neq k}}^{\operatorname{deg}(D)} D_{i} s^{i}}{D_{k} s^{k}} u-\frac{N_{1}(s)}{D_{k} s^{k}} u+\frac{1}{D_{k} s^{k}} \sum_{j}\left(N_{2 j}(s)+N_{3 j}(s) Y_{q}\right) I_{j} \\
\left(s^{2}+\frac{R_{q q}}{L_{q q}} s+\omega_{q}^{2}\right) v=-\left(s^{2}+\frac{R_{q q}}{L_{q q}} s+\omega_{q}^{2}\right) \frac{\sum_{\substack{\operatorname{deg}\left(D^{\prime}\right) \\
i \neq k}}^{\prime} D_{i}^{\prime} s^{i}}{D_{k}^{\prime} s^{k}} v-\frac{N_{1}^{\prime}(s)}{D_{k}^{\prime} s^{k}} v+\frac{1}{D_{k}^{\prime} s^{k}} \sum_{j}\left(N_{2 j}^{\prime}(s)+N_{3 j}^{\prime}(s) Y_{q}\right) I_{j}
\end{array}\right.
$$

System (24) takes the form (25). Since $\left(D(s), N_{1}(s), N_{2 j}(s), N_{3 j}(s)\right)$ and $\left(D(s), N_{2}^{\prime}(s), N_{2 j}^{\prime}(s), N_{3 j}^{\prime}(s)\right)$ are polynomials in the $s$ variable, the 2 equations of the system (24) are of the form (25).

$$
\left\{\begin{array}{l}
\left(s^{2}+\omega_{q}^{2}\right) u=-\frac{R_{q q}}{L_{q q}} s u-\left(s^{2}+\frac{R_{q q}}{L_{q q}} s+\omega_{q}^{2}\right) \frac{\sum_{\substack{i=0 \\
i \neq k}}^{\operatorname{deg}(D)} D_{i} s^{i}}{D_{k} s^{k}} u-\frac{N_{1}(p)}{D_{k} p^{k}} u+\frac{1}{D_{k} s^{k}} \sum_{j}\left(N_{2 j}(s)+N_{3 j}(s) Y_{q}\right) I_{j} \\
\left(s^{2}+\omega_{q}^{2}\right) v=-\frac{R_{q q}}{L_{q q}} v v-\left(s^{2}+\frac{R_{q q}}{L_{q q}} s+\omega_{q}^{2}\right) \frac{\sum_{\substack{i=0 \\
i \neq k}}^{\operatorname{deg}\left(D^{\prime}\right)} D_{i}^{\prime} s^{i}}{D_{k}^{\prime} s^{k}} v-\frac{N_{1}^{\prime}(p)}{D_{k}^{\prime} p^{k}} v+\frac{1}{D_{k}^{\prime} s^{k}} \sum_{j}\left(N_{2 j}^{\prime}(s)+N_{3 j}^{\prime}(s) Y_{q}\right) I_{j}
\end{array}\right.
$$

The previous system is of the form (26), this ODE describes the oscillator behavior and can be solved by asymptotic method. Even in the case of quite simple oscillator, the coefficients $a$ and $b$ may have a fairly tedious expansion.

$$
\left\{\begin{array}{l}
\left(s^{2}+\omega_{q}^{2}\right) u=\sum_{i} a^{i} s^{i} u+\sum_{j=1 \ldots n} \sum_{i} b_{j}^{i} s_{j}^{i} I_{j} \\
\left(s^{2}+\omega_{q}^{2}\right) v=\sum_{i} a^{i} s^{i} v+\sum_{j=1 \ldots n} \sum_{i} b_{j}^{i} s_{j}^{i} I_{j}
\end{array}\right.
$$

\section{Conclusion}

We have proposed an algorithm to obtain the ordinary differential equation of the oscillator behavior, including the individual noise sources. All the equations necessary for this computation are detailed.

The use of formal calculus allows us to fully automate this analysis. The software we have developed parses the oscillator input netlist, identifies and performs the topological reduction as well as all computations of the equation.

Moreover, the formal calculus approach offers a great flexibility because it can be decided which variable must be replaced by its numerical value or which one should be maintained in its literal form.

Numerical resolution of the obtained nonlinear ODE by using asymptotic method allows us to obtain oscillator steady state features, transient signal signal as well as amplitude and phase noise spectra [3]. So, this program gives the user an efficient and powerful analysis tool for quartz crystal oscillator design.

\section{Acknowledgments}

This work has been supported by CNES and DGA under contracts: \#832/CNES/88/5374/00, \# 962/CNES/91/1476/00, \# 962/CNES/94/1230/00.

\section{References}

[1] R. Brendel, F. Djian, E. Robert, High precision nonlinear computer modelling techniques for quartz crystal oscillators, Proc. 45th AFCS (1991).

[2] R. Brendel, G. Marianneau, T. Blin, M. Brunet, Computer aided design of quartz crystal oscillators, IEEE Trans. on UFFC, vol. 42, n. 4, pp. 700-708 (1995).

[3] R. Brendel, N. Ratier, L. Couteleau, G. Marianneau, P. Guillemot, Nonlinear analysis of noise in quartz crystal oscillators, Proc. of 12 th EFTF (1998).

[4] M.E. Frerking, Crystal oscillator design and temperature compensation, Van Nostrand Reinhold Co (1978). 
[5] L.W. Nagel, SPICE 2 : A computer program to simulate semiconductor circuits, Memorandum ERL-M520, Univ. of California, Berkeley (1975).

[6] N. Ratier, R. Brendel, T. Blin, G. Marianneau, P. Guillemot, Non linear simulation of quartz crystal oscillators, Proc. of 9th EFTF, pp. 8892, (1995).

[7] N. Ratier, R. Brendel, P. Guillemot, Quartz oscillators: deriving oscillation condition by sym- bolic calculus, Proc. of 10th EFTF, pp. 442-446, (1996).

[8] N. Ratier, L. Couteleau, R. Brendel, P. Guillemot, Automatic formal derivation of the oscillation condition, Proc. of 51st IFCS, pp. 925-931, (1997).

[9] P. Tuinenga, SPICE - A guide to circuit simulation and analysis using PSpice, 2nd edition, Prentice Hall, 1992. 